\title{
Discussion on the Possibility of Metal Foil in Chinese Green Painting
}

\author{
Xiangziyou ${ }^{1}$ \\ ${ }^{1}$ College of Fine Arts, Hunan Normal University, Changsha, Hunan, 410012, China \\ 1271674590@qq.com
}

\begin{abstract}
Exploring the likelihood of metal foil in the blue-green mountain water painting, verifying its use by actual examples. The likelihood of metal foil is used in Qingli Mountain Water Paintings, which has great significance for the innovation of green mountain water, and has a driving effect on traditional paintings inheritance and innovation.
\end{abstract}

Keywords: metal foil, Chinese painting, Chinese green painting

\section{RESEARCH SIGNIFICANCE}

Chinese art and foreign art collision is increasingly fierce, traditional Chinese paintings have been greatly affected, and the materials of Chinese paintings have gradually enriched. For the in-depth study of Dunhuang Mural and Temple Buddha Sticker, coupled with traditional paintings and innovation, making contemporary Chinese painters using metal foil. The literati paintings for foil, and even the soul of green mountain water is because they think this gorgeous color is very common, but the responsibility is not in the nature of the foil, but because the ancients give him its right, rich, money symbol, make foil The performance in the picture is covered by people. The metal foil is reused to Chinese paintings, but also the innovation and breakthrough of painting materials, but also explains that contemporary painters re-emphasis on my country's traditional culture, wanting to develop our traditional paintings better, and more accurate, closer to Chinese painting The direction of development. The topics I studied in practice are also to enable themselves to examine the use of foil in the green mountain water in a correct concept. It can understand the foil in painting in the painting, the use of the foil. Strong art effect. I will show a part of the foil in the green mountain water by my own practice, and I hope that my experiment will help me or other students' painting creation.

\section{APPLICATION OF METAL FOIL IN PAINTING}

The metal foil is widely used in sculptures and religious murals, and the use of foil makes the picture more gorgeous and rich. Religious murals also appear to be lightweight because of metal foil. The metal foil is not only used in sculptures and murals, but also began to use metal pigments into Chinese painting in the Tang Dynasty. LiSixun is very good at using metallic materials in the Chinese green Painting. He has created the big green painting and JinBi painting, and the green painting has been developed. His representative painted "Jiang Fan Dynasty".

The use of foil in contemporary painting is also more and more, many people study the innovative use of metal foil in painting. Zhang Daqian's blue-green painting style is a variety of varieties. On the basis of the green, you will use the golden to hit the rock, the shaded stone is mixed with gold. "Xianyincezhang painting" is one of the representatives of the contemporary JinBi painting, this painting is like the fire, the mountain stone is endless.

\section{POSSIBLE USE OF METAL FOIL IN CHINESE GREEN PAINTING}

Different metal foils can be used according to the painter's conception, creativity, and color, and can also determine how the method and techniques of the use of metal foil can be determined according to the picture effect of the painter to achieve. For example, if our screen needs to be shiny and the overall metal foil, we can put a whole metal foil to the picture; if we need to have a matte effect, we can use a hard pen to attach the whole Block foil is grinding; if we need local sticking foil, we can only apply glue on the needs of the need, tear down the extra foil after sticking; if you want to achieve special, natural texture, you can Burning foil, 
there will be some picture effects that use the pigment during the firing foil; or you can put the foil on the paper or cloth, then polished or fired, and then see the picture effect at this time. The speacial Chinese painting creation

\subsection{Research significance}

Chinese art and foreign art collisions are increasingly fiercely, traditional Chinese paintings have been greatly affected, and the materials of Chinese painting have gradually enriched. For the in-depth study of Dunhuang Mural and Temple Buddha Sticker, coupled with traditional paintings and innovation, making contemporary Chinese painters using metal foil. The literati paintings for foil, and even the soul of green mountain water is because they think this gorgeous color is very common, but the responsibility is not in the nature of the foil, but because the ancients give him its right, rich, money symbol, make foil The performance in the picture is covered by people. The metal foil is reused to Chinese paintings, but also the innovation and breakthrough of painting materials, but also explains that contemporary painters re-emphasis on my country's traditional culture, wanting to develop our traditional paintings better, and more accurate, closer to Chinese painting The direction of development. The topics I studied in practice are also to enable themselves to examine the use of foil in the green mountain water in a correct concept. It can understand the foil in painting in the painting, the use of the foil. Strong art effect. I will show a part of the foil in the green mountain water by my own practice, and I hope that my experiment will help me or other students' painting creation.

\subsection{Personality in the green mountain water}

\subsubsection{Possibility preset and actual operation}

The foil has unlimited use in the Chinese green painting, and some use methods have been opened up by the former. In contemporary, accompanied by the continuous integration of Chinese and Western culture and continuous collision, the integrated materials are gradually applied to Chinese paintings. The painters continue to develop new painting materials in different materials. Everything in life can express some ideas. Even can only play the decorative role, making things more intense to be used to painting. Painting materials are not limited to pen ink paper, pigments, etc. can be said that the use of materials, only can't be used, no need to use. I mainly introduce several possibilities I can imagine the possibility of the foil in the green mountain water, the use of foil in painting is infinitely possible, and the change in foil is random and infinite, it is not expected.
The foil can be replaced in a color in the picture, which further processes the green mountain water, that is, the places where the special effect is needed, this painting is a terraced terraces, and some mountain stone trees have been added around. Terraced fields are also created by painters, Chinese painters also like to use ink to show terraces. In this picture of the terraces, it is a terraces in the form of green mountain water. Finally, the copper foil is posted on the field. The color of the copper foil itself has a little bit orange, and the color of the soil is relatively close, so even in the field The copper foil is posted, and it will not be very awkward, and the decorative is enhanced, the copper foil has its metal bright attribute, so that the decoration effect of the picture is more intense. If you think that the copper foil appears to be dead in the screen, you can vividly vivid; it can also be polished on the top of the copper foil on the top, and it is also a variety of ways to grind, for example, can be used with rough and mozzle, can be used The sandpaper is grinding, it is necessary to pay attention to the wear process must be careful, do not wait for the gelatin to dry, we must grind when it is dry, and the foil does not be polished. Summary The first method is that the foil can replace some color in the Chinese green painting, which seems to have a lot of color and decorative, and it has a breakthrough in material use, which seems to have innovative. What foil is needed according to your own picture, because different foils have different colors.

The foil can be smashed after being decorated in the picture, the foil has a high gloss, and the mashed foil does not even be evenly even as gold powder, there will be different shapes of foil, which will make the picture more vivid. It is also possible to carry out a burnt foil according to the picture effect you want to achieve, the silver foil is more likely to be oxidized than copper foil and gold foil. Silver will have some blue, yellow, these colors are not formulated during the burnfaction, and the colors in the green mountain water also need breakthrough and innovation, so this method of burning foil can be applied to the green mountain water. Make it more in the picture to be more natural. Depending on the desired picture, the foil can be used as the background, and the foil is integrated or partially foil on the paper, and the foil can also be used as a rock, and some adjustment improvements in the screen.

The foil has an unlimited possible application in the green mountain water waiting for us to discover, innovate, and break through the tradition. Qinglong Mountain has developed bottlenecks in the literati painting period, because the literati painters think that the color of the green mountainous mountain is too bright, and people feel that there is not so elegant. However, the painting of the green mountain water and literati is not the same style. The use of foil in the green mountain water is also waiting for us to open up innovation, but the literati painters give the Qingloushan 
water, leading to the development of the green mountain water. The use of foil in Chinese green painting has also been developed. With the continuous collision of the Chinese and Western culture, the painters have begun to re-examine the status of Qinglongshan water in China, and the green mountain water is resended, and it has also been developed. The green mountain water itself is a treasure in China's landscape painting, which has a very important position in China's landscape painting, and is the source of the development of landscape paintings, so we should carry forward the treasures of green mountain water.

\subsubsection{Precautions in the experiment}

Can't be painted in the foil, and the coat will destroy the shape you want to post, because it will cause the glue flow to the area outside the brush, but destroy the shape; After the rubber dried, the excess foil was torn, and the glue did not dry when torn, and it will tear incomplete, it will form a waste; if you need to polish the close foil, this When it is completely dry, wait until 78 , you can use a hard brush to polish it. Because the foil is completely sticky on the paper, it will not be able to grind it; when burning foil, pay attention to keep indoor ventilation, Because the gas generated after the sulfur powder is heated, it is necessary to wear a mask; also pay attention to wearing a mask when the foil is particularly soft, easy to break, even the breath can also cause the foil deformed, so It is best to wear a mask when foil.

\subsection{Practical conclusion}

According to practice, the foil has played a rich role in the picture effect of the Chinese green painting and many accidents that have not been reached with pigments. Especially when burning foils, some chemical reactions occurred, and these chemical reactions usually the picture effect is occasional, which is that we can't expect in advance. This kind of blue-green and brown and black, which have occurred due to chemical reactions, is the color of the pigment that cannot be adjusted, even if it can be adjusted, but there is no chemical reaction to the chemical reaction of the foil and the sulfur powder. Natural, accidental Tiancheng. According to part practices, the use of foil in the green mountain water is very broad, and there is unlimited possibilities, it is necessary to continuously practice in future painting creation, constantly summed up, and then get more perfect, more comprehensive theoretical system.

\section{THE USE OF METAL FOIL IN ITS OWN GREEN MOUNTAINOUS WATER CREATION}

The use of metal foil in the green mountain water in the green mountain water has enhanced the performance of the green mountainous work, and the metal foil also as a historic integrated painting material, green mountain water as a treasure of Chinese painting. Combine with metal foil perfect. General Tang Dynasty painter's size Li Jin combines foil with green mountain water, producing Jin Bishan water, and Jin Bishan water has gradually developed, and the development of mountain water painting has greatly promoted. Now, with the Chinese and Western paintings continue to communicate, collide, the metal foil has brought our different visual experience, so more and more painters will study and explore the foil in green when they are created by green mountain water. Different uses in landscape. The use of the foil in the green mountain water should be more rich and diverse, because the gold participates in the formation of Jin Bishan water in the green mountain water, which has made the green mountain water in China's landscape paintings. In the creation of green mountain water, I decided to use silver foil into creation. The color after the silver foil is very rich, and some places will become a very blue green, and some will become dark gray, which naturally has a very wonderful color due to chemical reactions. The combination of green and silver foil and silver foil is more able to highlight the green mountain water is noble, gorgeous and elegant qualities, silver foil is slightly simple, making the picture harmony and richness, and the rich level. My creation uses silver foil and copper foil. In creation, the silver foil is sticker in the foot of the mountain and then the silver foil is firing, and the blue-green or black gray is present after the silver foil, so that the screen has a feeling of cloud. The other is to attach the copper foil in the top of the mountainhead after the overtilving stone trees, and then use hard to play this kind of spots.

\section{CONCLUSION}

The use of foil in the green mountain water has only a long and far-reaching history, and it is constantly developing in contemporary, its techniques and mode of use are constantly being updated, constantly created. The metal foil is used in the green mountain water, not just in the innovation of the painting material, is also the unique artistic charm of JinBi painting in the Tang Dynasty. The decorative effect of the foil is also a bit of paint, and the influence of Buddhism's Chinese water painting has led to the introduction of Qinglv painting.It is a very sacred and noble color. Any golden thing is beautiful and sacred in their eyes. Therefore, the application of metal foil in painting should also be further developed, rather than being given a ruthenium, we should inherit and develop traditional things and develop in traditional basis. I believe that under our common efforts, Chinese traditional culture will develop better and better. 


\section{REFERENCES}

[1] Liu Yue. Metal foil used in contemporary painting [M]. Education Art, 2013.

[2] Zhang Xiaolun. On the Intervention of Integrated Materials in Contemporary Chinese Painting [D]. Northwest Normal University, 2014.

[3] Li Jianzhen. Study on the performance of metal materials in Chinese painting [J]. Shenyang: Shenyang Normal University, 2011.

[4] Li Xizhi. Application and exploration of metal foil in modern heavy color painting $[\mathrm{J}]$. Shandong: Volkswagen, 2010.

[5] Zhang Gui. Modern Heavy Color Painting Metal Foil Performance Techniques [M]. Guangzhou: Lingnan Art Publishing House, 2003.

[6] Chen Yanfeng. Application of integrated materials in China [J]. Guangzhou: Art Life Artistic Concept, 2014.

[7] Wang Zhenzhen, Chen Yaoming. Artistic Performance of Integrated Materials [M]. Shanghai: Shanghai University Press, 2005.

[8] Li Shiping. New Vision of Comprehensive Painting Materials [M]. Hubei Science and Technology Press, 2009.4. 